\title{
Polarization Effects in Lambda and Anti-Lambda Production at HERMES
}

S. L. Belostotski, and HERMES collaboration

Citation: AIP Conference Proceedings 1149, 664 (2009); doi: 10.1063/1.3215733

View online: https://doi.org/10.1063/1.3215733

View Table of Contents: http://aip.scitation.org/toc/apc/1149/1

Published by the American Institute of Physics

\section{Conference Proceedings}

\section{Get $30 \%$ off all print proceedings!}

Enter Promotion Code PDF30 at check 


\title{
Polarization Effects in Lambda and Anti- Lambda Production at HERMES
}

\author{
S.L. Belostotski on behalf of the HERMES collaboration ${ }^{\mathrm{a}}$ \\ ${ }^{a}$ Petersburg Nuclear Physics institute, PNPI 188300 Gatchina, Lenigrad district, Russia
}

\begin{abstract}
Study of the $\Lambda$ and $\Lambda$ hyperon production in the HERMES experiment is discussed. The data were collected using a $27.6 \mathrm{GeV}$ longitudinally polarized positron (or electron) beam of the HERA accelerator and a polarized or unpolarized target. The transverse polarizations (perpendicular to the production plane) of the $\Lambda$ and $\Lambda$ hyperons have been measured. The spin transfer $K_{L L}$ from the longitudinally polarized target has been found for inclusively detected $\Lambda$ and $\Lambda$ events. Longitudinal spin transfer $D_{L L}$ from the beam to the $\Lambda$ hyperon in the deep inelastic scattering regime has been analyzed. The $D_{L L}$ for $\Lambda$ has been measured for the first time.
\end{abstract}

Keywords: lambda, polarization.

PACS: $13.88 .+\mathrm{e}, 13.60 .-\mathrm{r}, 13.60 . \mathrm{Rj}$.

\section{INTRODUCTION}

Given the nontrivial spin structure of the proton, it is of interest to consider the spin structure of other baryons. In this respect the $\Lambda$ hyperon, as the lightest strange baryon of the SU(3) spin-1/2 octet, is particularly interesting. The HERMES experiment [1] has studied $\Lambda$ and $\Lambda$ electro and photo-production using polarized positron (electron) beam and polarized or unpolarized targets. In these experiments, the most important observable is polarization of the produced hyperon which is accessed through the parity violating weak decay channel $\Lambda^{0} \rightarrow \mathrm{p} \pi^{-}\left(\bar{\Lambda}^{0} \rightarrow \overline{\mathrm{p}} \pi^{+}\right)$.

The results on transverse ("spontaneous") $\Lambda(\Lambda)$ polarization in quasi-real photoproduction and on longitudinal spin transfer $D_{L L}$, to the $\Lambda$ from the beam in semi-inclusive Deep Inelastic Scattering (DIS) based on a limited number of events corresponding to HERMES RUN I (the years 1996-2000) have already been published by the HERMES collaboration [2-4]. Recently, HERMES has reported on measurement of the spin transfer $K_{L L}$ to the $\Lambda(\Lambda)$ hyperon from the longitudinally polarized target [5]. Here we present the data on $D_{L L}$ obtained in HERMES RUN I and RUN II during the years 1996-2000 and 2002-2005, respectively, analyzed jointly. A recently developed method of $D_{L L}$ extraction which does not require exact balance of the positive and negative helicity in the primary beam has been used. In the previous analysis, only unpolarized target data was analyzed. In this analysis, polarized target data has also been included under condition that the frequently flipped target polarization is well helicity balanced which corresponds to average zero polarization.

CP1149, Spin Physics, $18^{\text {th }}$ International Spin Physics Symposium

edited by D. G. Crabb, D. B. Day, S. Liuti, X. Zheng, M. Poelker, and Y. Prok

(C) 2009 American Institute of Physics 978-0-7354-0686-5/09/\$25.00 
Finally, the gain in statistics of a factor of 4 has been obtained which allows one to better study $D_{L L}$ kinematical distributions and to extract for the first time $D_{L L}$ for $\Lambda$.

\section{LONGITUDINAL SPIN TRANSFER $D_{L L}$}

The longitudinal spin transfer $D_{L L}$, to the $\Lambda$ hyperon was studied by the LEP experiments OPAL and ALEPH at an energy corresponding to the $Z_{0}$ pole [6,7]. In these experiments the hyperons were predominantly produced via the strange quark anti-quark decay channel in which the primary strange quarks initiating the hadronization process were negatively polarized at the level of $91 \%$. The OPAL and ALEPH (LEP) data show a significant polarization transfer from the $s(\bar{s})$ quark to the $\Lambda(\Lambda)$ hyperon, thus confirming that the $s$ quark dominates the spin of $\Lambda$ hyperon. In contrast to the LEP experiments, the production of $\Lambda$ hyperons in DIS is originated predominately from the struck $u$ or $d$ quark emitted from the target nucleon, the contribution from the strange quark being on average small. The spin transfer coefficient $D_{L L^{\prime}}$ describes the probability that the polarization of the struck quark is transferred to the $\Lambda$ hyperon along the secondary quantization axis $L^{\prime}$ in the $\Lambda$ rest frame. In this analysis, the quantization axis $L^{\prime}$ is chosen along the direction of the $\Lambda$ momentum (as it is not affected by the relativistic transformation to the $\Lambda$ rest frame). Another possible choice of the spin transfer direction is $L^{\prime}=L$, i.e. along the spin of the virtual photon. Note that for HERMES kinematics the angle between the virtual photon momentum (spin) and $\Lambda$ momentum in the $\Lambda$ rest frame is not small, i.e., unlike the case of very high energies, $L$ and $L^{\prime}$ are not collinear. In principle, the spin transfer may have two components in the $\Lambda$-production plane: longitudinal one, along the virtual photon spin $(L)$, and transverse one, perpendicular to this direction, and $L^{\prime}$ axis (direction of the spin transfer) does not necessarily aligned with the $\Lambda$ momentum. An analysis with the two components of the spin transfer reconstructed independently is now in progress, while here in this report it is assumed that $L^{\prime}$ in the $\Lambda$ rest frame is along the direction of $\Lambda$ momentum.

The $D_{L L}$, has been extracted using a data sample consisting of $26714 \Lambda$ events and $3610 \Lambda$ events with the restriction $Q^{2}>0.8 \mathrm{GeV}$ applied, where $Q^{2}$ is the negative four-momentum transfer squared. In order to cancel the spectrometer acceptance effect, the $D_{L L}$, has been determined by combining the two data sets measured with opposite beam polarizations [8]. It is important that in this approach the extracted $D_{L L^{\prime}}$ does not contain an uncertainty coming from the Monte Carlo simulation of the spectrometer acceptance.

As the HERMES spectrometer is a forward detector, the Feynman variable $x_{F}$ for $\Lambda$ production is mostly positive ranging from -0.25 to 1 . The $x_{F}>0$ requirement cuts off only a small fraction of events. The spin-transfer coefficient for $\Lambda$ averaged within full $x_{F}$ range is found to be $D_{L L^{\prime}}=0.102 \pm 0.056_{\text {stat }} \pm 0.02_{\text {syst }}$. With the requirement

$x_{F}>0, \quad D_{L L^{\prime}}=0.082 \pm 0.061_{\text {stat }} \pm 0.02_{\text {syst }}$. For the $\Lambda$ in full $x_{F}$ range it is found $D_{L L^{\prime}}=-0.040 \pm 0.112_{\text {stat }} \pm 0.03_{\text {syst }}$. As $\Lambda$ cannot be produced from a target remnant 
mechanism, in this case the cut on $x_{F}$ has not been applied. The HERMES results on $D_{L L^{\prime}}$ versus $x_{F}$ for $\Lambda$ are presented in Fig. 1 (left panel) together with the results of NOMAD[9] and COMPASS[10] experiments at CERN and E665[11] at FNAL. Two points for the $\Lambda$ and $Q^{2}$ dependence for the $\Lambda$ are shown in Fig. 1 (right panel). Most of NOMAD data have been obtained at negative $x_{F}$. For positive $x_{F}$, all existing to date experimental results indicate that the $D_{L L^{\prime}}$ is compatible with zero within the statistical uncertainty about \pm 0.10 . In principle, the observation of a small (or equal to zero) value of $D_{L L}$, points to the dominance of scattering from $u$ or $d$ quarks whose polarization within the $\Lambda$ hyperons is small. Note that recent lattice-QCD calculations find small light quark polarizations in the $\Lambda$ hyperon: $\Delta u^{\Lambda}=\Delta d^{\Lambda}=-0.02 \pm 0.04$ and $\Delta s^{\Lambda}=0.68 \pm 0.04[12]$.
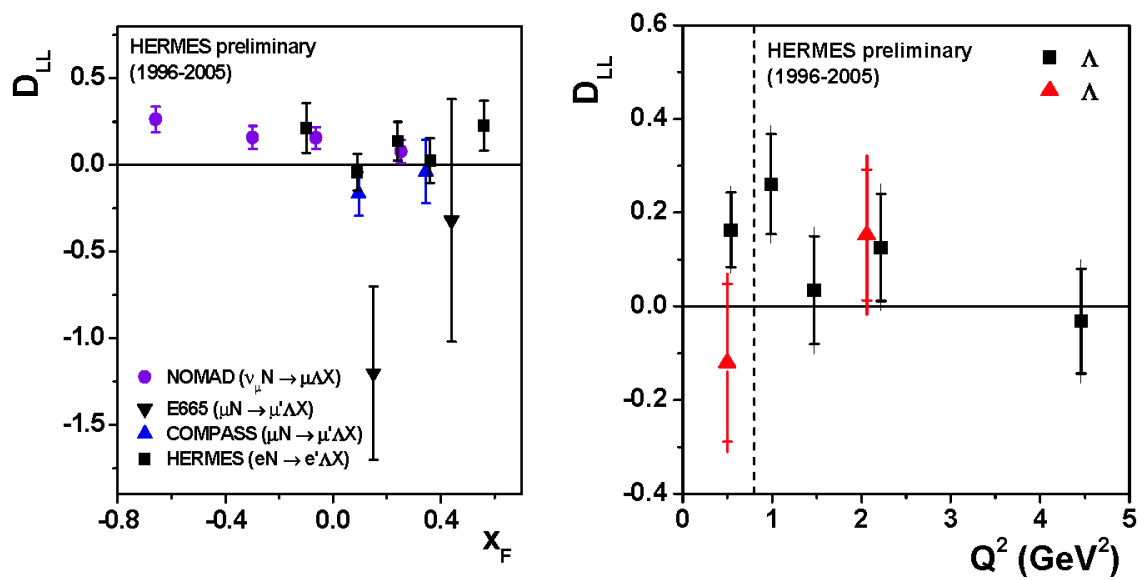

FIGURE 1. Longitudinal spin transfer from the beam to the $\Lambda$ versus $x_{F}$ (left panel) together with available world data. Longitudinal spin transfer from the beam to the $\Lambda$ and $\Lambda$ versus $Q^{2}$ (right panel).

The vertical dashed line is positioned at $Q^{2}=0.8 \mathrm{GeV}^{2}$. Two points for $\Lambda$ are averaged results for $\mathrm{Q}^{2}<0.8 \mathrm{GeV}^{2}$ and $\mathrm{Q}^{2}>0.8 \mathrm{GeV}^{2}$ domains, respectively.

On the other hand, the condition $\Delta u^{\Lambda}=\Delta d^{\Lambda} \equiv 0$ does not necessarily mean that the $D_{L L^{\prime}}$ vanishes everywhere. Thus, authors [13] have employed a phenomenological model to explore the dependences of $D_{L L^{\prime}}$ on kinematical variables. They predict increasing positive values of the spin transfer with $D_{L L^{\prime}} \rightarrow 1$ at $x_{F} \rightarrow 1$ $\left(z=E^{\Lambda} / \nu \rightarrow 1\right)$.

Unfortunately, interpretation of the spin transfer coefficient is not straightforward. For a $\Lambda$ produced in DIS regime via a current quark $q$ the "partial" spin-transfer from a quark $q$ can be expressed as $D_{L L^{\prime}}^{q \Lambda}=\frac{\Delta F^{q \Lambda}}{F^{q \Lambda}}$, where $\Delta F^{q \Lambda}$ and $F^{q \Lambda}$ is spin-dependent and spin-independent $q \rightarrow \Lambda$ fragmentation function, respectively. If a fractional contribution from a quark $q$ (purity) is $\omega_{q} \quad\left(\sum_{q} \omega_{q}=1\right)$ the total spin transfer is given 
by $D_{L L^{\prime}}=\sum_{q} \omega_{q} D_{L L^{\prime}}^{q \Lambda}$. Taking into account $u$-dominance, a naïve expectation of $D_{L L^{\prime}}$ in DIS is $D_{L L^{\prime}} \approx D_{L L^{\prime}}^{u \Lambda}=\frac{\Delta F^{u \Lambda}}{F^{u \Lambda}} \approx \frac{\Delta u^{\Lambda}}{u^{\Lambda}}$. One should remember, however, that at the moderate energy of the HERMES experiment the distinction between the current and target fragmentation domain is not very sharp and $\Lambda$ fragmentation may be affected by the target remnant mechanism even at positive $x_{F}$. Further, the fraction of $\Lambda$ hyperons produced via heavier hyperon decays is significant: only about $40 \%$ of the $\Lambda$ hyperons are produced directly from the string fragmentation. The contribution from heavier hyperons $\Sigma^{0}, \Sigma^{*}$ or $\Xi$, which may decay into the polarized $\Lambda$ hyperons and thus modify the $D_{L L^{\prime}}$, must be taken into account.

\section{ACKNOWLEDGMENTS}

I gratefully acknowledge Yu. Naryshkin and D. Veretennikov for their essential contribution to the analysis of the presented experimental data.

\section{REFERENCES}

1. HERMES Collaboration, K. Ackerstaff et al., Nucl. Instrum. Methods A 417, 230 (1998).

2. HERMES Collaboration, A. Airapetian et al., Phys. Rev. D 76, 092008 (2007).

3. HERMES Collaboration, A. Airapetian et al., Phys. Rev. D 74, 072004 (2006).

4. HERMES Collaboration, A. Airapetian et al., Phys. Rev. D 64, 112005 (2001).

5. D. Veretennikov in Proceedings of the XVI International Workshop on Deep-Inelastic Scattering and Related Topics (spin physics), London, England, April 7-11, (2008).

6. OPAL Collaboration, K. Ackerstaff et al., Eur. Phys. J. C 2, 49 (1998).

7. ALEPH Collaboration, D. Buskulic et al., Phys. Lett. B 374, 319 (1996).

8. S. Belostotski in Proceedings of the IX-Workshop on High Energy Spin Physics (SPIN-01), Dubna2001, preprint LINR E1,2-2002-103, 192 (2002).

9. NOMAD Collaboration, P. Astier et al., Nucl. Phys. B 588, 3 (2000).

10. COMPASS Collaboration, M. Sapozhnikov (private communication).

11. E665 Collaboration, M. R. Adams et al., Eur. Thys. J. C 17, 263 (2000).

12. OSDSF Collaboration, M. Göckeler et al., Phys. Rev. Lett. B 545, 112 (2002).

13. B.-Q. Ma, I. Schmidt, J. Soffer, and J.-J. Yang, Phys. Rev. D 65, 034004 (2002). 